\title{
Mild poikilocapnic hypoxia increases very low frequency haemoglobin oxygenation oscillations in prefrontal cortex
}

\author{
Agnieszka Gruszecka' ${ }^{1}$ Monika Waskow ${ }^{2}$, Marta A. Malkiewicz ${ }^{3,4}$, J. Patrick Neary ${ }^{5}$, Jyotpal Singh", \\ Taylor Teckchandani ${ }^{5}$, Gregory P. Kratzig ${ }^{6}$, Magdalena Wszedybyl-Winklewska ${ }^{7}$, Andrzej F. Frydrychowski ${ }^{8}$, \\ Jacek Rumiński ${ }^{9}$, Natalia Głowacka ${ }^{9}$, Piotr Lass ${ }^{10}$, Pawel J. Winklewski ${ }^{7}$ and Marcin Gruszecki ${ }^{1,9^{*}}$ (D)
}

\begin{abstract}
Background: The aim of the study was to investigate the effect of mild cerebral hypoxia on haemoglobin oxygenation $\left(\mathrm{HbO}_{2}\right)$, cerebrospinal fluid dynamics and cardiovascular physiology. To achieve this goal, four signals were recorded simultaneously: blood pressure, heart rate / electrocardiogram, $\mathrm{HbO}_{2}$ from right hemisphere and changes of subarachnoid space (SAS) width from left hemisphere. Signals were registered from 30 healthy, young participants (2 females and 28 males, body mass index $=24.5 \pm 2.3 \mathrm{~kg} / \mathrm{m}^{2}$, age $30.8 \pm 13.4$ years).

Results: We analysed the recorded signals using wavelet transform and phase coherence. We demonstrated for the first time that in healthy subjects exposed to mild poikilokapnic hypoxia there were increases in very low frequency $\mathrm{HbO}_{2}$ oscillations $(<0.052 \mathrm{~Hz})$ in prefrontal cortex. Additionally, SAS fluctuation diminished in the whole frequency range which could be explained by brain oedema.

Conclusions: Consequently the study provides insight into mechanisms governing brain response to a mild hypoxic challenge. Our study supports the notion that $\mathrm{HbO}_{2}$ and SAS width monitoring might be beneficial for patients with acute lung disease.
\end{abstract}

Keywords: Hypoxia, Wavelet transform, Blood pressure, Subarachnoid space width, Near infrared spectroscopy

\section{Introduction}

Hypoxia is frequently observed in a number of clinical situations such as lung disease, sleep apnoea, as well as cardiac and cerebrovascular dysfunction associated with aging. Mild hypoxia is also an eminent feature of the respiratory distress syndrome evoked by the coronavirus (SARS-CoV-2): severe acute respiratory syndrome (SARS). A number of cognitive and neuropsychiatric manifestations of SARS have been observed in a proportion of patients such as depressed mood, anxiety,

*Correspondence: mgruszecki@gumed.edu.pl

1 Department of Radiology Informatics and Statistics, Medical University of Gdansk, Tuwima Str. 15, 80-210, Gdansk, Poland

Full list of author information is available at the end of the article post-traumatic stress disorder and cognitive decline. Although these are multifactorial, changes in cerebral oxygen supply and related pathophysiological consequences may play a role [1].

It is well-known that hypoxia leads to a decline in haemoglobin oxygenation $\left(\mathrm{HbO}_{2}\right)$ in cerebral cortex [2]. Diminished oxygen supply to the brain results in several compensatory mechanisms, which in turn trigger substantial changes in various aspects of brain functioning. Augmented cerebral blood flow [3] and cerebral blood volume [4,5] together with blood-brain barrier impairment [6] lead to discrete brain oedema $[4,5]$ and increases in intracranial pressure $[4,5]$. original author(s) and the source, provide a link to the Creative Commons licence, and indicate if changes were made. The images or other third party material in this article are included in the article's Creative Commons licence, unless indicated otherwise in a credit line to the material. If material is not included in the article's Creative Commons licence and your intended use is not permitted by statutory regulation or exceeds the permitted use, you will need to obtain permission directly from the copyright holder. To view a copy of this licence, visit http://creativecommons.org/licenses/by/4.0/. The Creative Commons Public Domain Dedication waiver (http://creativeco mmons.org/publicdomain/zero/1.0/) applies to the data made available in this article, unless otherwise stated in a credit line to the data. 
Brain hypoxia also results in a number of metabolic changes associated with an augmented cerebral metabolic rate of oxygen, lactate and glutamate concentrations accompanied by a diminished creatinine concentration. These metabolic alterations suggest higher neural activity and increased oxidative metabolism [7, 8]. Despite these adaptations cytotoxic cerebral oedema may develop and subsequently evolve into ionic cerebral oedema, thus increasing grey matter volume even if blood-brain barrier still remains intact [9].

Transcranial Doppler studies demonstrate that acute mild normobaric hypoxia $\left(15 \% \mathrm{O}_{2}\right)$ augments both blood pressure (BP) and cerebral blood flow velocity (measured in middle cerebral artery) oscillations in the very-lowfrequency range $(0.02-0.07 \mathrm{~Hz})$, while does not affect $\mathrm{BP}$ and cerebral blood flow velocity fluctuations at cardiac and respiratory frequencies. These changes (at very low frequency), associated with increase in coherence and transfer function gain were typically explained by weakened autoregulatory control $[10,11]$.

The autoregulation dogma is however constantly challenged with increasing emphasis on either local neural or metabolic mechanisms $[12,13]$. The role of intracranial pressure in the feedback loops regulating widely understood cerebral metabolic homeostasis is continuously highlighted $[13,14]$. Consequently, in this study we aimed at assessing the effect of hypoxia on the oscillatory behaviour of BP, oxygenated haemoglobin $\left(\mathrm{HbO}_{2}\right)$, electrocardiogram (HR/ECG), and subarachnoid space (SAS) width using established non-invasive methods $[15,16]$.

The relative concentrations of the oxygenated and deoxygenated haemoglobin can be measured in the prefrontal area with near infrared spectroscopy (NIRS) [15]. Oscillatory changes in the SAS width, reflecting cerebrospinal fluid pulsatility [16], can be instantly monitored with the method developed by our team, called near-infrared transillumination-backscattering sounding (NIR-T/BSS) [16-19]. Both methods can be used simultaneously [20]. Wavelet transform analysis of biological signals can elegantly quantify and delineate the investigated interactions in both frequency and time domains $[12,21]$. The method is ideal to analyse oscillations of biological signals and was used many times by different groups.

Another useful mathematical tool to analyse biological signals are coupling functions which describe very precisely any interactions between considered systems. The coupling functions reveal information about the coupling strength but also are helpful to find the mechanism responsible for considered interactions. They determine the possibility of qualitative transitions between the oscillations e.g. routes into, and out of, phase synchronization. They are very suitable for studying interaction of oscillatory processes in physiology e.g., cardiorespiratory or cardiovascular interactions [22, 23]. Recently, Gruszecka et al. [24] studied the respiratory-cardiac coupling of the blood pressure and cerebrospinal fluid signals. Another interesting study was carried out by [25]. Combined wavelet transform and coupling functions helped to consider an interactions between three oscillations: respiratory, cardiac and vascular activity. The oscillations were extracted from three different signals: respiratory effort signal, electrocardiogram and laser Doppler flowmetry. Another example is that general anaesthesia can lead to important changes in the forms of coupling function between brain waves [26]. The detailed review about coupling functions and its application can be found in Stankovski et al. [27].

We hypothesized that mild hypoxia would increase the prominence of very low-frequency $\mathrm{HbO}_{2}$ local brain oscillations while maintaining the impact of central oscillatory components generated by the heart and lungs. We also expected SAS oscillations to diminish in the whole frequency range as a result of simulated altitude at approximately $2900-3000 \mathrm{~m}$.

\section{Results}

Four signals were continuously and simultaneously recorded from the volunteers: BP, HR/ECG, $\mathrm{HbO}_{2}$ RIGHT and $\mathrm{SAS}_{\text {LEFT }}$ while breathing normal room air $\left(19.8 \% \mathrm{O}_{2}\right)$, and during normobaric hypoxia $\left(14.8 \% \mathrm{O}_{2}\right)$ in an environmental chamber to simulate altitude at $2900-3000 \mathrm{~m}$. The atmospheric pressure for whole time of experiment was kept at the same level $712 \mathrm{mmHg}$, while the level of oxygen during normobaric hypoxia was reduced to $14.8 \%$. A gas mixture of $19.8 \% \mathrm{O}_{2}$ was used as the normoxic condition as this is the native partial pressure of oxygen in Regina, Saskatchewan, Canada (577 m above sea level) where the testing was performed. According to nonparametric Wilcoxon rank sum test (see Table 1) there was a statistically significant change of all measured signals during mild normobaric hypoxia.

Figure 1 shows the result of the amplitude of the wavelet transform (WT) for one representative volunteer. Left and right columns of Fig. 1 corresponds to breathing a gas mixture with $19.8 \%$ and $14.8 \% \mathrm{O}_{2}$, respectively. We estimated WT for all four measured signals: BP (a, e), HR/ECG (b, f), $\mathrm{HbO}_{2 \text { RIGHT }}(\mathrm{c}, \mathrm{g})$ and $\operatorname{SAS}_{\text {LEFT }}(\mathrm{d}, \mathrm{h}$ ).

To simplify the comparison between two stages of experimental procedure in terms of their frequency content, we plotted the median of the time-averaged amplitude of wavelet transforms (Fig. 2).

Figure 3 illustrates the wavelet phase coherence (left column) and phase difference (right column) between pairs of all collected signals. The value of phase coherence was significant when the value of phase coherence 
Table 1 Subject characteristics during breathing a gas mixture with $19.8 \%$ and $14.8 \% \mathrm{O}_{2}$

\begin{tabular}{|c|c|c|}
\hline & $\begin{array}{l}\text { Gas mixture with } 19.8 \% \\
\mathrm{O}_{2}\end{array}$ & $\begin{array}{l}\text { Gas mixture } \\
\text { with } 14.8 \% \\
\mathrm{O}_{2}\end{array}$ \\
\hline HR (beats/min) & $60.87_{68.41}^{54.34}$ & $66.41_{75.75}^{57.38^{* * *}}$ \\
\hline $\mathrm{DBP}(\mathrm{mmHg})$ & $65.45_{70.74}^{59.55}$ & $69.36_{74.26}^{62.56^{*}}$ \\
\hline $\mathrm{SBP}(\mathrm{mmHg})$ & $113.98_{120.18}^{103.98}$ & $119.63_{129.35}^{110.63^{* * *}}$ \\
\hline $\mathrm{MAP}(\mathrm{mmHg})$ & $82.09_{88.35}^{74.77}$ & $87.51_{91.84}^{79.91^{*}}$ \\
\hline $\operatorname{SAS}_{\text {LEFT }}(A U)$ & $0.19_{0.25}^{0.15}$ & $0.17_{0.23}^{0.14^{*}}$ \\
\hline $\mathrm{HbO}_{2 \text { RIGHT }}\left(\% \mathrm{O}_{2}\right)$ & $0.71_{0.87}^{0.54}$ & $0.52_{0.56}^{0.36 * *}$ \\
\hline $\mathrm{tHb}(\mu \mathrm{M})$ & $9.28_{11.36}^{7.55}$ & $14.76_{16.64}^{13.02 * *}$ \\
\hline $\mathrm{SaO}_{2}\left(\% \mathrm{O}_{2}\right)$ & $98_{99}^{97}$ & $92_{94}^{91 * * *}$ \\
\hline $\mathrm{EtCO}_{2}(\mathrm{mmHg})$ & $33.11_{35.17}^{31.63}$ & $31.59_{33.75}^{30.05^{* * *}}$ \\
\hline $\mathrm{EtO}_{2}(\mathrm{mmHg})$ & $127.65_{130.43}^{124.54}$ & $96.52_{99.78}^{94.25^{* * *}}$ \\
\hline
\end{tabular}

Values shown are median $n_{75 \text { th percentiles }}^{25 \text { thes }} \mathrm{p}$ values were estimated between two stages of the experimental procedure

$H R$ heart rate; $D B P$ diastolic blood pressure; $S B P$ systolic blood pressure; $M A P$ mean arterial pressure, $S A S_{\text {LEFT }}$ subarachnoid width; $\mathrm{HbO}_{2 \mathrm{RIGHT}}$ relative changes in oxyhaemoglobin; $t \mathrm{Hb}$ relative changes in total haemoglobin; $\mathrm{SaO}_{2}$ oxyhaemoglobin saturation; $\mathrm{EtCO}_{2}$ end-tidal $\mathrm{CO}_{2} ; \mathrm{EtO}_{2}$ end-tidal $\mathrm{O}_{2}$

${ }^{*} \mathrm{p}<0.05$; ${ }^{* *} \mathrm{p}<0.01$; ${ }^{* * *} \mathrm{p}<0.001$

was higher than 95th percentile of 435 (2-permutation of 30 subjects) inter-subject surrogate. When we observed statistically significant phase coherence at certain frequencies, we assumed the same for phase differences. For phase coherence we found statistically significant

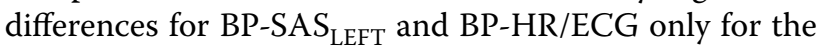
cardiac interval. We did not observe any statistically significant difference for phase difference for all considered pairs of signals.

\section{Discussion}

The novel findings of our study showed that mild poikilocapnic hypoxia increases the very low frequency $\mathrm{HbO}_{2}$ oscillations $(<0.052 \mathrm{~Hz})$ in prefrontal cortex while diminishing SAS oscillations in the whole frequency range. In addition we confirmed that prefrontal cortex $\mathrm{HbO}_{2}$ and SAS width decline during exposure to lowered oxygen partial pressure.

In recent years there has been an increased interest in cerebrovascular mechanisms related to normal and pathological physiology. As such, we have witnessed a substantial paradigm shift in our thinking in regard to brain perfusion control mechanisms. The increasing accumulation of evidence suggests that astrocytes act as physiological sensors reacting to changes in the brain parenchymal levels of metabolic substrates (such as oxygen), cerebral perfusion and intracranial pressure changes $[13,28-30]$. In particular, astrocytes might be responsible for local cellular signalling mechanisms adjusting cerebral perfusion to brain metabolic needs [13].

Our study strongly supports the view that local brain mechanisms, rather than systemic control-level mechanisms are responsible for adaptation to mild poikilocapnic (normoxic) hypoxia. We observed decreases in respiratory contribution to $\mathrm{HbO}_{2}$ prefrontal cortex oscillations, while at the same time observing substantially increased very low frequency $\mathrm{HbO}_{2}$ oscillations $(<0.052 \mathrm{~Hz})$. Very low frequency periodic dynamism is believed to reflect neural sympathetic and metabolic activity [12, 31], and is most likely linked to increased neural metabolism [7].

Prefrontal cortex $\mathrm{HbO}_{2}$ fluctuations at the $0.145-$ $0.052 \mathrm{~Hz}$ frequency were reported to be linked to general BP oscillations [32-34]. In response to normobaric hypoxia, BP fluctuations did not change in the 0.1450.052 frequency. We also did not observe any phase difference in the $\mathrm{HbO}_{2}$ and BP signals. Stabilisation of BP-brain signals oscillations at the $0.145-0.052 \mathrm{~Hz}$ frequency are most likely driven by increased sympathetic activity related to hypoxia and slightly increased intracranial pressure $[4,5,35]$. We have previously shown that high sympathetic drive strengthen the links between BP and SAS signals [14].

Slightly increased intracranial pressure and discrete brain swelling previously reported in hypoxia could have resulted in a decline of SAS oscillations across the whole frequency range. Although we cannot confirm discrete brain swelling from our study, our findings extend previous results collected in our laboratory where healthy subjects were exposed to 5 min of poikilokapnic (normobaric) hypoxia, and we observed a trend towards a decline in SAS width and diminished BP-SAS amplitude coupling [36]. In the current study, we observed diminished BP-SAS and HR/ECG-BP phase coherences within the cardiac frequency band. Suppression of respiratory SAS oscillations were reported previously by our team as an early indicator of rising intracranial pressure in rabbit [18]. A decline in the modulation of cerebral blood flow by even moderately increased intracranial pressure was also observed in humans [37].

This study used poikilocapnic (normobaric) hypoxia to challenge whether $\mathrm{EtCO}_{2}$ fluctuations varied in response to reflexive hyperventilation. We preferred this over an isocapnic hypoxic challenge, defined by hypoxia in the presence of maintained $\mathrm{CO}_{2}$ within the bloodstream, as we believe our chosen methodology better mimicked clinical conditions. A gas mixture of $19.8 \% \mathrm{O}_{2}$ was used as the normoxic condition as this is the native partial pressure of oxygen in Regina, Saskatchewan, Canada (577 $\mathrm{m}$ above sea level) where the testing was performed. 


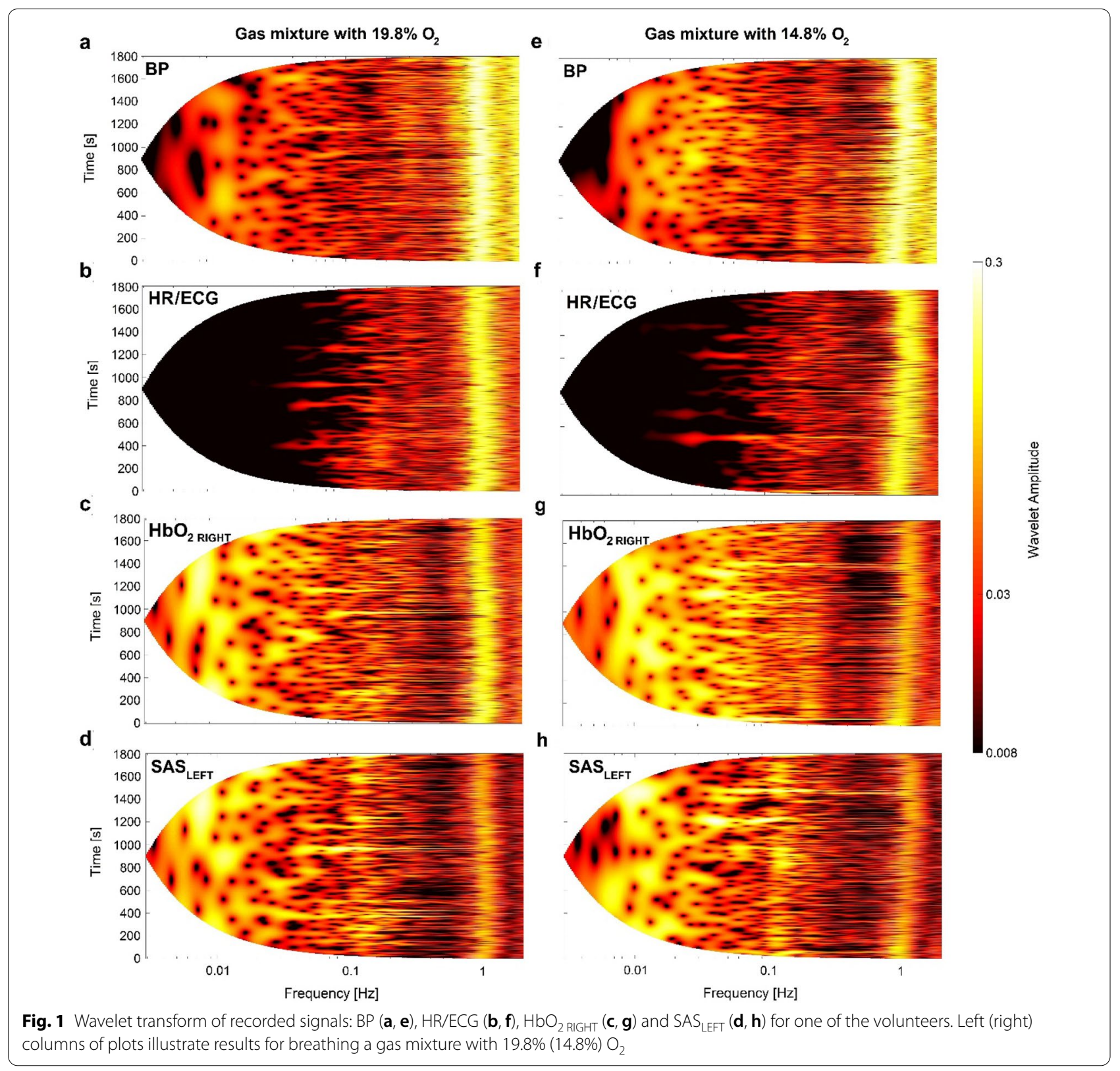

Our study demonstrates that clinical situations associated with even mild cerebral hypoxia may result in several pathophysiological adaptations including changes in brain metabolism and cerebrospinal fluid dynamics. Non-invasive modalities that enable continuous brain monitoring could provide several benefits to patients suffering from diseases associated with declined brain oxygenation, stroke or traumatic brain injury.

\section{Conclusions}

In subjects exposed to mild poikilokapnic hypoxia we have demonstrated for the first time that: (1) very low
$\mathrm{HbO}_{2}$ oscillations $(<0.052 \mathrm{~Hz})$ increase in prefrontal cortex, and (2) SAS fluctuation diminishes in the whole frequency range. Consequently the study provides insights into the physiological mechanisms governing brain response to a mild hypoxic challenge. Our study supports the notion that $\mathrm{HbO}_{2}$ and SAS width monitoring might be beneficial for patients with acute lung disease.

\section{Materials and methods Subjects}

A group of 30 healthy and not smoking volunteers (28 males and 2 females, age $30.8 \pm 13.4$ years, BMI 


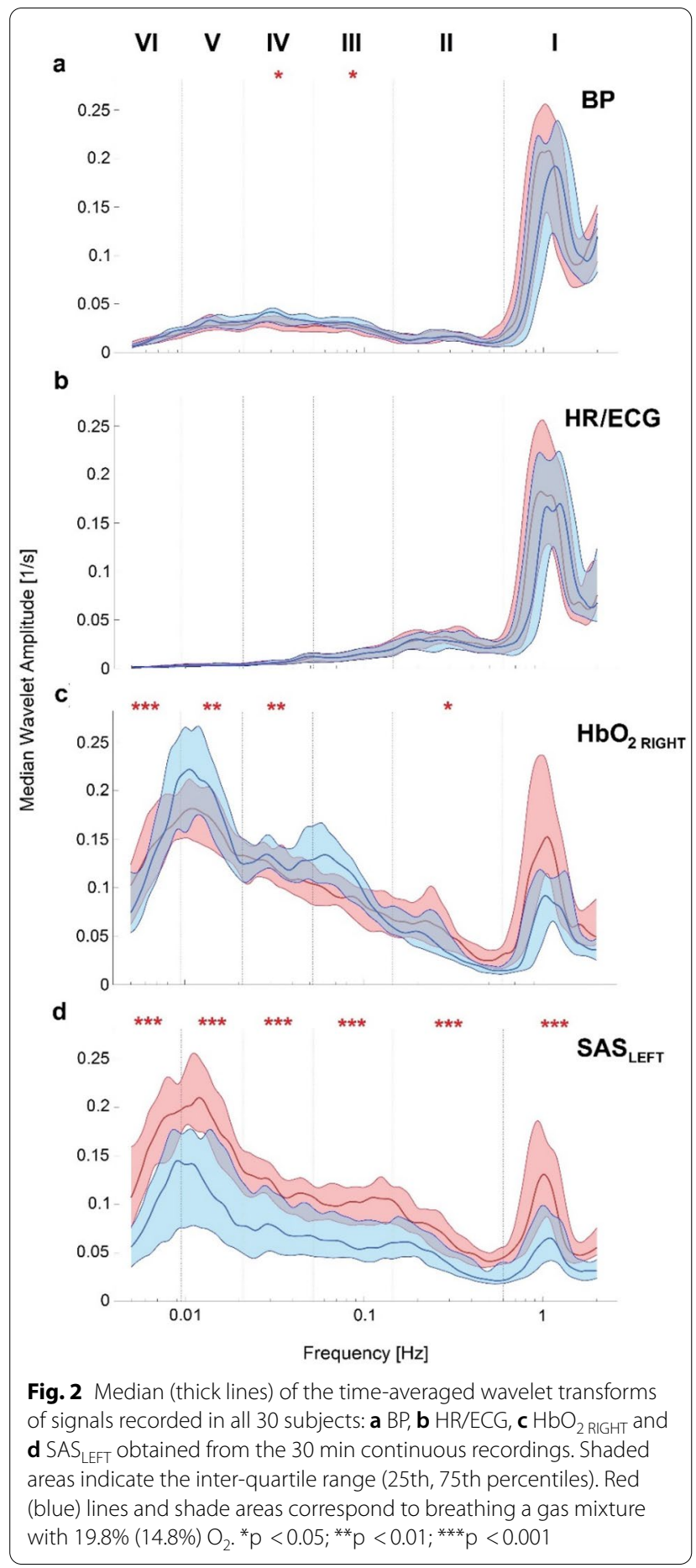

$=24.5 \pm 2.3 \mathrm{~kg} / \mathrm{m}^{2}$ ) were involved in the experiments, which were carried out in accordance with the recommendations of Helsinki. The Ethics Committee of University of Regina (REB\#2017-013) approved this study and the experimental protocol. All subjects were older than 18 years and signed a consent form to participate in the study. Participants were asked to avoid any alcohol at least $24 \mathrm{~h}$ before the experiment, and tea, coffee, nicotine, cocoa and any food and beverages containing methylxanthine for at least $12 \mathrm{~h}$ before the experiment. Intense exercise training was not allowed at least $6 \mathrm{~h}$ prior to testing, and all subjects were asked to void their bladder within $30 \mathrm{~min}$ of testing. All procedures were preceded by $10 \mathrm{~min}$ of rest in the sitting position in a comfortable chair located in quiet room.

\section{Experimental design}

Two tests of breathing a gas mixture with $19.8 \%$ and $14.8 \%$ $\mathrm{O}_{2}$ were conducted in a quiet room and environmental chamber. Room and chamber were pre-set to a temperature of $18-20{ }^{\circ} \mathrm{C}$. After all of the medical research equipment was attached to the volunteer participant (see "Measurements" Section below), they were instructed to lie supine on a bed with a pillow to support their head during breathing a gas mixture of normal room air $\left(19.8 \% \mathrm{O}_{2}\right)$ and during normobaric hypoxia $\left(14.8 \% \mathrm{O}_{2}\right)$. The first $30 \mathrm{~min}$ of experiment, subjects were asked to breath room air $(19.8 \%$ $\mathrm{O}_{2}$ ), and then they moved to the environmental chamber with the aid of a research assistant (it took about 3-5 min). During the next $30 \mathrm{~min}$, volunteer subjects breathed freely in the hypoxic chamber with reduced levels of oxygen (14.8\%). The hypoxic oxygen condition mimiced the elevation of approximately 2900-3000 m above sea level.

\section{Measurements}

A Finometer (Finapres Medical Systems, Arnhem, The Netherlands) was used to measure BP. Finger photoplethysmography BP was calibrated against brachial arterial pressure. The signal was collected from the left middle finger at all times during both normoxia $\left(19.8 \% \mathrm{O}_{2}\right)$ and hypoxia $\left(14.8 \% \mathrm{O}_{2}\right)$. HR was determined from an ECG signal. The ECG ground electrode was placed on the left anterior superior iliac spine and the two main leads under the middle portion of each clavicle (Lead I) [20]. $\mathrm{SaO}_{2}$ was measured using a Nellcor PM10N Portable $\mathrm{SpO}_{2}$ Patient Monitoring System (Medtronic Canada, Vancouver, BC). During the experiment, the device was placed on the right index finger.

(See figure on next page.)

Fig. 3 Median (thick lines) of wavelet phase coherence (left column) and phase difference (right column) between a, $\mathbf{g} B P$ VS $S A S_{L E F T}, \mathbf{b}, \mathbf{h} B P$ VS

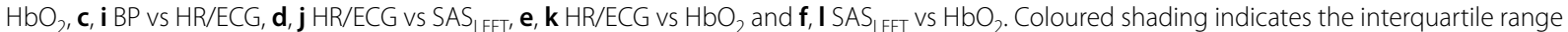
(25th, 75th percentiles) for 30 subjects. Coherence below the 95th percentile of the surrogates (light grey line and shading) is not considered significant. Red (blue) lines and shade areas correspond to breathing a gas mixture with 19.8\% (14.8\%) $\mathrm{O}_{2} \cdot{ }^{*} \mathrm{p}<0.05 ;{ }^{* *} p<0.01 ;{ }^{* * *} p<0.001$ 


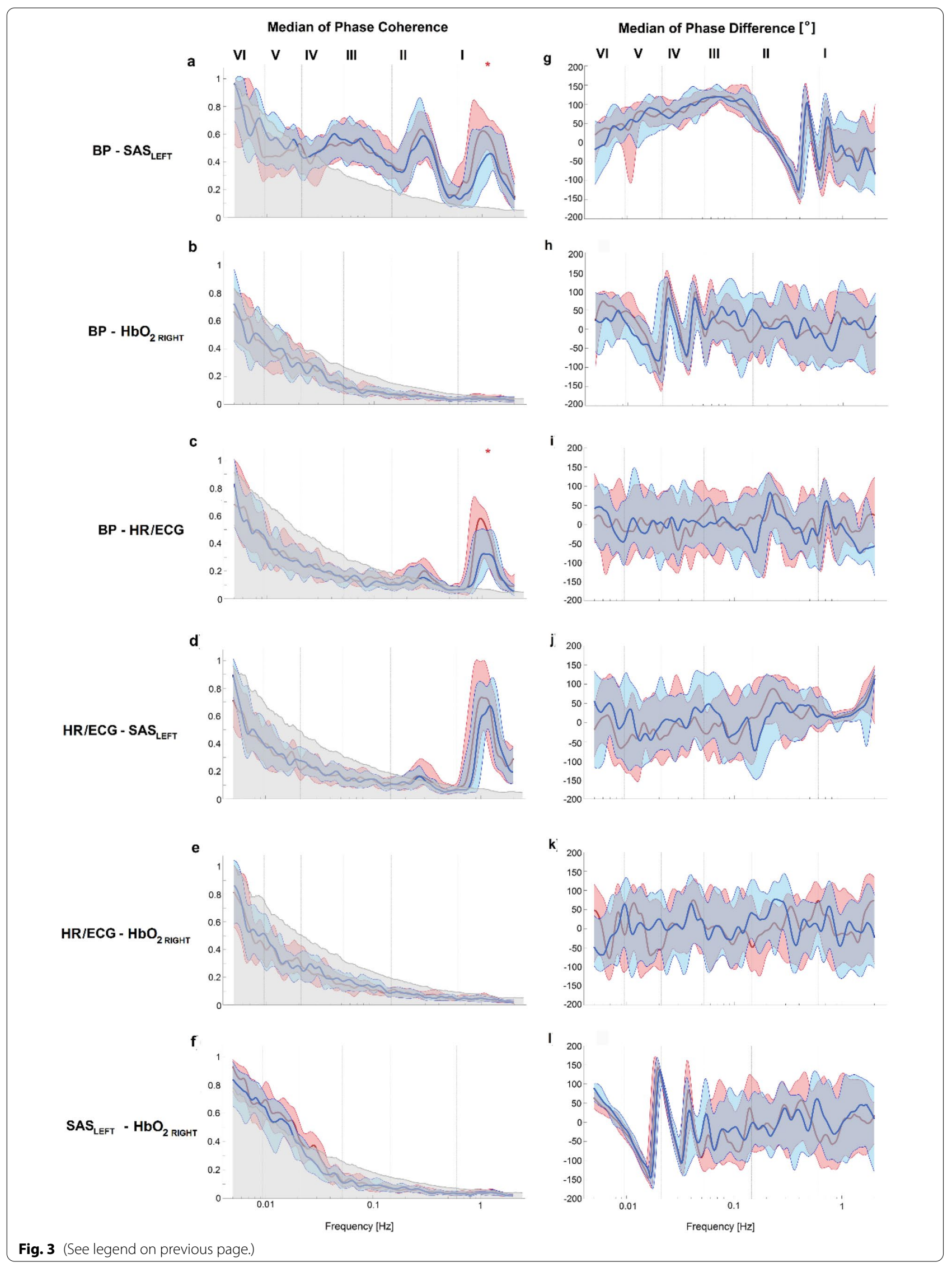


Expired respiratory gas samples from the mouthpiece were constantly analysed using the side-stream technique for $\mathrm{EtCO}_{2}$ using AD Instruments ML206 gas analyser (Colorado Springs, USA). SAS signal was collected using an SAS Monitor (NIRTI SA, Wierzbice, Poland). A detailed description of the SAS Monitor has been provided previously (e.g., [16]. The NIRS signal was collected by PortaLite system (Artinis Medical, The Netherlands). The sensor was positioned over pre-frontal cortex of the right hemisphere during the experiment. NIRS contains transmitters at 30,35 and $40 \mathrm{~mm}$ from the receiver, which allows a penetration depth of approximately one-third to one-half of the distance between optodes [38]. During the experiment, we collected the following NIRS signals: relative changes in oxy- $\left(\mathrm{HbO}_{2}\right)$, deoxy- $(\mathrm{HHb})$, total haemoglobin $\left(\mathrm{tHb}=\mathrm{HbO}_{2}+\mathrm{HHb}\right)$ and haemoglobin difference $\left(\mathrm{Hb}_{\text {diff }}=\mathrm{HbO}_{2}-\mathrm{HHb}\right)$. To avoid admission of background light the NIRS sensor was secured with a black coloured tensor bandage. We decided to analyse a NIRS signal that penetrate the deepest regions of head to be sure that we truly analyse signals from brain vessels. Like many others, [39-42] we focused our analyses on the oxyhaemoglobin $\left(\mathrm{HbO}_{2}\right)$ signal. Additionally, to avoid any interference between NIRS and SAS signals, we recorded only SAS $_{\text {LEFT }}$ from left hemisphere and $\mathrm{HbO}_{2}$ RIGHT from right hemisphere.

All parameters were recorded and saved simultaneously for further analysis. This was completed for both segments, with the first 30 min normoxic condition completed first, followed by the subsequent 30 min normobaric hypoxic second. To import and view collected signals, a PowerLab 8/32 amplifier was coupled with LabChart 7 Pro (AD Instruments, Colorado Springs, Colorado, USA). All signals before analysis were down sampled to $10 \mathrm{~Hz}$, detrended using a moving average with a window size of $220 \mathrm{~s}$, and normalized by subtraction of their mean and division by their standard deviation.

\section{Wavelet transform}

To detect and perform analysis of physiological processes that are responsible for generating oscillations in the cardiovascular system, we used wavelet analysis. The wavelet transform is a method that transforms a signal from the time domain to the time-frequency domain. The definition of the wavelet transform is:

$$
W(s, t)=\frac{1}{\sqrt{s}} \int_{-\infty}^{+\infty} \varphi\left(\frac{u-t}{s}\right) g(u) d u,
$$

where $W(s, t)$ is the wavelet coefficient, $g(u)$ is the time series and $\varphi$ is the Morlet mother wavelet, scaled by factor $s$ and translated in time by $t$. The Morlet mother wavelet is defined by the equation:

$$
\varphi(u)=\frac{1}{\sqrt[4]{\pi}} \exp (-i 2 \pi u) \exp \left(-0.5 u^{2}\right)
$$

where $i=\sqrt{-1}$. The rationale for using the Morlet wavelet is that it affords good localization of events in time and frequency due to its Gaussian shape [39, 42]. The wavelet coefficients are complex numbers in the timefrequency plane when the Morlet wavelet is used:

$$
X\left(\omega_{k}, t_{n}\right)=X_{k, n}=a_{k, n}+i b_{k, n} .
$$

They define the instantaneous relative phase,

$$
\theta_{k, n}=\arctan \left(\frac{b_{k, n}}{a_{k, n}}\right)
$$

and the absolute amplitude,

$$
\left|X_{k, n}\right|=\sqrt{a_{k, n}^{2}+b_{k, n}^{2}},
$$

for each frequency and time.

During the measurement, hypoxia may create phase modulations. A mathematical tool to find the relationship between the phases of two signals is the wavelet phase coherence (WPCO). WPCO enables us to determine whether the oscillations detected are significantly correlated over time. To estimate the WPCO we used the following expression [43]:

$$
C_{\theta}\left(f_{k}\right)=\frac{1}{n}\left|\sum_{t=1}^{n} \exp \left[i\left(\theta_{2 k, n}-\theta_{1 k, n}\right)\right]\right|,
$$

where $\theta_{k, n}=\arctan \left(\frac{b_{k, n}}{a_{k, n}}\right)$ is an instantaneous measure of phases at each time $t_{n}$ and frequency $f_{k}$ for both signals. When two oscillations are unrelated (related), their phase difference continuously changes (remain constant) with time, thus their $C_{\theta}\left(f_{k}\right)$ approaches zero (one).

Additionally, we can calculate the phase difference $\Delta \theta_{k}$, which provides information about the phase lag of one oscillator compared to the other:

$$
\Delta \theta_{k}=\arctan \left(\frac{\frac{1}{n} \sum_{t=1}^{n} \sin \left(\Delta \theta_{2 k, n}-\Delta \theta_{1 k, n}\right)}{\frac{1}{n} \sum_{t=1}^{n} \cos \left(\Delta \theta_{2 k, n}-\Delta \theta_{1 k, n}\right)}\right),
$$

where $\Delta \theta_{k} \in\left(-180^{\circ}, 180^{\circ}\right)$.

\section{Statistical analysis}

To avoid the assumption of normality in the results, nonparametric statistical tests were used for all comparisons. The Wilcoxon rank sum test was used to compare whether the median of results for breathing a gas mixture with $19.8 \% \mathrm{O}_{2}$ and $14.8 \% \mathrm{O}_{2}$ was significantly 
different. The results of our calculations are found in Table 1; Figs. 2, 3.

Our analysis were supported by added six different frequency intervals which correspond to different physiological functions described previously by Stefanovska et al. [21] and Gruszecki et al. [16] (see Figs. 2, 3). Additionally, we estimated the $\mathrm{p}$ value of the differences between the results for breathing a gas mixture with $19.8 \%$ and $14.8 \% \mathrm{O}_{2}$ for all measured signals. We observed the highest differences $(p<0.001)$ for WT amplitude for SAS $S_{\text {LEFT }}$ for all frequency intervals. For $\mathrm{HbO}_{2}$ RIGHT the differences between amplitude of wavelet transform were associated with respiration (II, 0.145-0.6 Hz), neurogenic (IV, 0.021-0.052 Hz), endothelial nitric oxide (NO) dependent (V, 0.00950.021 ) and NO independent (VI, 0.005-0.0095 Hz). In turn, WT amplitude of the BP signal only has differences $(\mathrm{p}<0.05)$ in myogenic (III, $0.052-0.145 \mathrm{~Hz}$ ) and neurogenic (IV) intervals. We did not observe any statistically significant differences for the HR/ECG signal.

To test whether the estimated values of phase coherence are statistically significant, the surrogate data testing method was used [44]. As we know there are naturally less cycles of oscillations the lower in frequency that we consider. This can cause artificially increased wavelet phase coherence at low frequencies, even in cases where there is none. The surrogate analysis helps us to find a significance level above which the phase coherence may be regarded as physically meaningful. To estimate significance level, we used intersubject surrogates [45], which assumes that the signals collected from different subjects must be independent, while having similar characteristic properties. For 30 volunteers we used 435 intersubject surrogates. The actual value of phase coherence obtained at each frequency can then be compared with the surrogate threshold. When the phase coherence is located above the threshold it is considered to be statistically significant.

\begin{abstract}
Abbreviations
$\mathrm{HbO}_{2}$ : Haemoglobin oxygenation; BP: Blood pressure; SAS: Subarachnoid space; WT: Wavelet transform; NIRS: Near infrared spectroscopy; HR: Heart rate; DBP: Diastolic blood pressure; SBP: Systolic blood pressure; MAP: Mean arterial pressure; $\mathrm{tHb}$ : Relative changes in total haemoglobin; $\mathrm{O}_{2}$ : Oxygen; $\mathrm{SaO}_{2}$ : Oxyhaemoglobin saturation; $\mathrm{EtCO}_{2}$ : End-tidal $\mathrm{CO}_{2} ; \mathrm{EtO}_{2}$ : End-tidal $\mathrm{O}_{2} ; \mathrm{NO}$ : Nitric oxide; WPCO: Wavelet phase coherence.
\end{abstract}

\section{Acknowledgements}

We would like to thank all of the volunteer participants that contributed their valuable time to these experiments.

\section{Authors' contributions}

Conceived and designed the experiments: AG, MG, JPN, PJW. Performed the experiments: JS, TT, MG. Analysed the data: AG, MG. Contributed reagents/ materials/analysis tools: MAM, PN, GPK, MWW, AF, JR, NG, AG, PL, MG. Co-wrote the paper: AG, MG, PJW. All authors read and approved the final manuscript.
Funding

Dr Marcin Gruszecki was supported by the NCN-Grant Number 2018/02/X/ NZ4/00464.

\section{Availability of data and materials}

The data presented in this study are available on request from the corresponding author. The data are not publicly available due to privacy.

\section{Declarations}

\section{Ethics approval and consent to participate}

The Ethics Committee of University of Regina (REB\#2017-013) approved this study and the experimental protocol. All subjects were older than 18 years and signed a consent form to participate in the study.

\section{Consent for publication}

All authors reviewed the manuscript and agreed to publish the paper.

\section{Competing interests}

Profs Andrzej F. Frydrychowski and Pawel J. Winklewski are stakeholders in NIRTI SA. The other authors declare no competing interests.

\section{Author details}

${ }^{1}$ Department of Radiology Informatics and Statistics, Medical University of Gdansk, Tuwima Str. 15, 80-210, Gdansk, Poland. ${ }^{2}$ Institute of Health Sciences, Pomeranian University of Slupsk, Slupsk, Poland. ${ }^{3}$ Department of Human Physiology, Applied Cognitive Neuroscience Lab, Medical University of Gdansk, Gdansk, Poland. ${ }^{4}$ Department of Psychiatry, Medical University of Gdansk, Gdansk, Poland. ${ }^{5}$ Faculty of Kinesiology and Health Studies, University of Regina, Regina, Canada. ${ }^{6}$ Department of Psychology, University of Regina, Regina, SK, Canada. ${ }^{7}$ Department of Human Physiology, Medical University of Gdansk, Gdansk, Poland. ${ }^{8}$ NIRTI SA, Wroclaw, Poland. ${ }^{9}$ Department of Biomedical Engineering, Faculty of Electronics, Telecommunications and Informatics, Gdansk University of Technology, Gdansk, Poland. ${ }^{10}$ Department of Nuclear Medicine, Medical University of Gdansk, Gdansk, Poland.

Received: 1 September 2021 Accepted: 30 November 2021

Published online: 14 December 2021

\section{References}

1. Alonso-Lana S, Marquié M, Ruiz A, Boada M. Cognitive and neuropsychiatric manifestations of COVID-19 and effects on elderly individuals with dementia. Front Aging Neurosci. 2020;12:588872.

2. Hampson NB, Camporesi EM, Stolp BW, Moon RE, Shook JE, Griebel JA, Piantadosi CA. Cerebral oxygen availability by NIR spectroscopy during transient hypoxia in humans. J Appl Physiol. 1990;69(3):907-13.

3. Kety SS, Schmidt CF. The effects of altered arterial tensions of carbon dioxide and oxygen on cerebral blood flow and cerebral oxygen consumption of normal young men. J Clin Invest. 1948;27(4):484-92.

4. Dubowitz DJ, Dyer EAW, Theilmann RJ, Buxton RB, Hopkins SR. Early brain swelling in acute hypoxia. J Appl Physiol. 2009;107:244-52.

5. DiPasquale DM, Muza SR, Gunn AM, Li Z, Zhang Q, Harris NS, Strangman GE. Evidence for cerebral edema, cerebral perfusion, and intracranial pressure elevations in acute mountain sickness. Brain Behav. 2016;6(3):e00437.

6. Halder SK, Milner R. Mild hypoxia triggers transient blood-brain barrier disruption: a fundamental protective role for microglia. Acta Neuropath Commun. 2020;8:175.

7. Vestergaard MB, Lindberg U, Aachmann-Andersen NJ, Lisbjerg K, Christensen SJ, Law I, Rasmussen P, Olsen NV, Larsson HBV. Acute hypoxia increases the cerebral metabolic rate - a magnetic resonance imaging study. J Cereb Blood Flow Metab. 2016;36(6):1046-58.

8. Vestergaard MB, Ghanizada H, Lindberg U, Arngrim N, Paulson OB, Gjedde A, Ashina M, Larsson HBW. Human cerebral perfusion, oxygen consumption, and lactate production in response to hypoxic exposure. Cereb Cortex. 2021. https://doi.org/10.1093/cercor/bhab294.

9. Biller A, Badde S, Heckel A, Guericke P, Bendszus M, Nagel AM, Heiland S, Mairbäurl H, Bärtsch P, Schommer K. Exposure to 16 h of normobaric 
hypoxia induces ionic edema in the healthy brain. Nat Commun. 2021;12:5987.

10. Iwasaki K, Ogawa Y, Shibata S, Aoki K. Acute exposure to normobaric mild hypoxia alters dynamic relationships between blood pressure and cerebral blood flow at very low frequency. J Cereb Blood Flow Metab. 2007;27(4):776-84.

11. Nishimura N, Iwasaki K, Ogawa Y, Aoki K. Decreased steady-state cerebral blood flow velocity and altered dynamic cerebral autoregulation during 5-h sustained 15\% $\mathrm{O}_{2}$ hypoxia. J Appl Physiol. 2010;108(5):1154-61.

12. Saleem S, Teal PD, Kleijn WB, Ainslie PN, Tzeng YC. Identification of human sympathetic neurovascular control using multivariate wavelet decomposition analysis. Am J Physiol Heart Circ Physiol. 2016;311 (3):H837-48.

13. Marina N, Christie IN, Korsak A, Doronin M, Brazhe A, Hosford PS, Wells JA, Sheikhbahaei S, Humoud I, Paton JFR, Lythgoe MF, Semyanov A, Kasparov S, Gourine AV. Astrocytes monitor cerebral perfusion and control systemic circulation to maintain brain blood flow. Nat Commun. 2020;11:131.

14. Winklewski PJ, Wolf J, Gruszecki M, Wszedybyl-Winklewska M, Narkiewicz K. Current understanding of the effects of inspiratory resistance on the interactions between systemic blood pressure, cerebral perfusion, intracranial pressure, and cerebrospinal fluid dynamics. J Appl Physiol. 2019;127:1206-14.

15. Ferrari $M$, Quaresima $V$. A brief review on the history of human functional near-infrared spectroscopy (fNIRS) development and fields of application. Neuroimage. 2012;63(2):921-35.

16. Gruszecki M, Nuckowska MK, Szarmach A, Radkowski M, Szalewska D, Waskow M, Szurowska E, Frydrychowski AF, Demkow U, Winklewski PJ. Oscillations of subarachnoid space width as a potential marker of cerebrospinal fluid pulsatility. Adv Exp Med Biol. 2018;1070:37-47.

17. Frydrychowski A, Gumiński W, Rojewski M, Kaczmarek JW, Juzwa W. Technical foundations for noninvasive assessment of changes in the width of the subarachnoid space with near-infrared transillumination-backscattering sounding (NIR-TBSS). IEEE Trans Biomed Eng. 2002;49(8):887-904.

18. Frydrychowski AF, Wszedybyl-Winklewska M, Guminski W, Przyborska A, Kaczmarek J, Winklewski PJ. Use of near infrared transillumination/back scattering sounding (NIR-T/BSS) to assess effects of elevated intracranial pressure on width of subarachnoid space and cerebrovascular pulsation in animals. Acta Neurobiol Exp. 2011;71(3):313-21.

19. Gruszecki M, Lancaster G, Stefanovska A, Neary JP, Dech RT, Guminski W, Frydrychowski AF, Kot J, Winklewski PJ. Human subarachnoid space width oscillations in the resting state. Sci Rep. 2018;8:3057.

20. Gruszecka A, Gruszecki M, Neary JP, Singh J, Teckchandani T, Waskow M, Wszedybyl-Winklewska M, Guminski W, Frydrychowski AF, Ruminski J, Lass P, Kratzig GP, Winklewski PJ. Comparison of near infrared spectroscopy (NIRS) and near-infrared transillumination-backscattering sounding (NIR-T/BSS) methods. Sci Rep. 2020;10(1):18668.

21. Stefanovska A, Bracic M, Kvernmo HD. Wavelet analysis of oscillations in the peripheral blood circulation measured by laser Doppler technique. IEEE Transact Biomed Eng. 1999;46(10):1230-9.

22. latsenko D, Bernjak A, Stankovski T, Shiogai Y, Owen-Lynch PJ, Clarkson PBM, McClintock PVE, Stefanovska A. Evolution of cardiorespiratory interactions with age. Phil Trans R Soc Lond A. 2013;371:20110622.

23. Lukarski D, Ginovska M, Spasevska H, Stankovski T. Time window determination for inference of time-varying dynamics application to cardiorespiratory interaction. Front Physiol. 2020;11:341.

24. Gruszecka A, Nuckowska M, Waskow M, Kot J, Winklewski P, Gumiński W, Frydrychowski AF, Wtorek J, Bujnowski A, Lass P, Stankovski T, Gruszecki M. Coupling between blood pressure and subarachnoid space width oscillations during slow breathing. Entropy. 2021;23(1):113.

25. Ticcinelli V, Stankovski T, latsenko D, Bernjak A, Bradbury A, Gallagher A, Clarkson PBM, McClintock PVE, Stefanovska A. Coherence and coupling functions reveal microvascular impairment in treated hypertension. Front Physiol. 2017:8:2.

26. Stankovski T, Petkoski S, Raeder J, Smith AF, McClintock PVE, Stefanovska A. Alterations in the coupling functions between cortical and cardio-respiratory oscillations due to anaesthesia with propofol and sevoflurane. Phil Trans R Soc A. 2016;374:20150186.

27. Stankovski T, Pereira T, McClintock PVE, Stefanovska A. Coupling functions: universal insights into dynamicalinteraction mechanisms. Rev Mod Phys. 2017:89:045001.

28. Angelova PR, Kasymov V, Christie I, Sheikhbahaei S, Turovsky E, Marina N, Korsak A, Zwicker J, Teschemacher AG, Ackland GL, Funk GD, Kasparov
S, Abramov AY, Gourine AV. Functional oxygen sensitivity of astrocytes. J Neurosci. 2015;35(29):10460-73.

29. Marina N, Turovsky E, Christie IN, Hosford PS, Hadjihambi A, Korsak A, Ang R, Mastitskaya S, Sheikhbahaei S, Theparambil SM, Gourine AV. Brain metabolic sensing and metabolic signaling at the level of an astrocyte. Glia. 2018;66(6):1185-99.

30. Turovsky EA, Braga A, Yu Y, Esteras N, Korsak A, Theparambil SM, Hadjihambi A, Hosford PS, Teschemacher AG, Marina N, Lythgoe MF, Haydon PG, Gourine AV. Mechanosensory signaling in astrocytes. J Neurosci. 2020;40(49):9364-71.

31. Chow N, Bell RD, Deane R, Streb JW, Chen J, Brooks A, Nostrand WV, Miano $J M$, Zlokovic BV. Serum response factor and myocardin mediate arterial hypercontractility and cerebral blood flow dysregulation in Alzheimer's phenotype. PNAS. 2007;104(3):823-8.

32. van Beek AHEA, Lagro J, Olde-Rikkert MGM, Zhang R, Claassen JAHR. Oscillations in cerebral blood flow and cortical oxygenation in Alzheimer's disease. Neurobiol Aging. 2012;33(2):428.e21-31.

33. Vermeij A, van den Abeelen ASSM, Kessels RPC, van Beek AHEA, Claassen JAHR. Very-low-frequency oscillations of cerebral hemodynamics and blood pressure are affected by aging and cognitive load. Neurolmage. 2014;85:608-15

34. Yunjie T, Blaise DF. Time lag dependent multimodal processing of concurrent fMRI and near-infrared spectroscopy (NIRS) data suggests a global circulatory origin for low-frequency oscillation signals in human brain. Neuroimage. 2010;53(2):553-64.

35. Talbot NP, Balanos GM, Dorrington KL, Robbins PA. Two temporal components within the human pulmonary vascular response to approximately $2 \mathrm{~h}$ of isocapnic hypoxia. J Appl Physiol. 2005;98:1125.

36. Wszedybyl-Winklewska M, Wolf J, Swierblewska E, Kunicka K, Gruszecka A Gruszecki M, Kucharska W, Winklewski PJ, Zabulewicz J, Gumiński W, Pietrewicz M, Frydrychowski AF, Bieniaszewski L, Narkiewicz K. Acute hypoxia diminishes the relationship between blood pressure and subarachnoid space width oscillations at the human cardiac frequency. PLoS ONE. 2017;12(2):1-13.

37. Haubrich C, Czosnyka Z, Lavinio A, Smielewski P, Diehl RR, Pickard JD, Czosnyka M. Is there a direct link between cerebrovascular activity and cerebrospinal fluid pressure-volume compensation? Stroke. 2007;38(10):2677-80.

38. Cui W, Kumar C, Chance B. Experimental study of migration depths for the photons measured at sample surface. SPIE. 1991;1431:180-91.

39. Bernjak A, Stefanovska A, McClintock PVE. Coherence between fluctuations in blood flow and oxygen saturation. Fluct Noise Lett. 2012;11:1-12.

40. Cui R, Zhang M, Li Z, Xin Q, Lu L, Zhou W, Han Q, Gao Y. Wavelet coherence analysis of spontaneous oscillations in cerebral tissue oxyhaemoglobin concentrations and arterial blood pressure in elderly subjects. Microvasc Res. 2014;93:14-20.

41. Kim TJ, Kim JM, Lee JS, Park SH, Jeong HB, Choi JK, Kim K, Bae HM, Ko SB. Prognostication of neurological outcome after cardiac arrest using wavelet phase coherence analysis of cerebral oxygen. Resuscitation. 2020;150:41-9.

42. Bernjak A, Stefanovska A, McClintock PVE, Owen-Lynch PJ, Clarkson P. Coherence between fluctuations in blood flow and oxygen saturation. Fluct Noise Lett. 2012;11:1-12.

43. Lachaux JP, Lutz A, Rudrauf D, Cosmelli D, Le Van QM, Martinerie J, Varela F. Estimating the time-course of coherence between single-trial brain signals: an introduction to wavelet coherence. Clin Neurophysiol. 2002;32:157.

44. Lancaster G, latsenko D, Pidde A, Ticcinelli V, Stefanovska A. Surrogate data for hypothesis testing of physical systems. Physics Rep. 2018;748:1-60.

45. Sun J, Hong X, Tong S. Phase synchronization analysis of EEG signals: an evaluation based on surrogate tests. IEEE Trans Biomed Eng. 2012;59:2254-63.

\section{Publisher's Note}

Springer Nature remains neutral with regard to jurisdictional claims in published maps and institutional affiliations. 